\title{
Calculation of Transients in a System of Algebraically Connected Dynamic Components Using a New Numerical Algorithm
}

\author{
ADAM SEMLYEN, SENIOR MEMBER, IEEE
}

\begin{abstract}
An efficient numerical algorithm is presented for the solution of transients in systems consisting of dynamic components with both slow and fast eigenvalues, interconnected at their terminals by an algebraic network.

The approach consists of producing algebraic input-output equivalents for the individual components by implicit integration of their differential equations. The adopted implicit integration is by parts leaving the impulse response intact and unaffected by the numerical discretization. The effect of this procedure is excellent convergence with large step sizes, resulting in significant savings in computation.

The method has been tested on a conveniently chusen large circuit and compared to alternative algorithms. It is successfully applied to the solution of power system transients problems (dynamic stability, switching transients). Although described for linear systems, the method can be applied for nonlinear systems, linearized after each step around the new operating point.
\end{abstract}

\section{INTRODUCTION}

A SPECIAL characteristic of many man-made systems is that dynamic components are interconnected by a nondynamic (algebraic) "network," in order to keep the resultant system as simple and manageable as possible. An example, among others, is an interconnected power system. The components (generators with their controls and prime connected to infinite power buses), have both slow and fast eigenvalues, in a synchronously rotating frame of reference. The latter are, however, short-lived (relatively high negative real parts): if attenuated or ignored, then the terminal voltages and currents can be described by slowly varying phasors, interrelated by the network admittance matrix, which represents clearly an algebraic relationship. However, strictly speaking, the network is also dynamic (consisting of $R, L, C$ components) and, consequently, the whole system is a very complex, dynamic conglomerate. In addition, switching operations (inception of short circuits, their clearing, reclosure of lines, etc.) make the problem timevarying; the fast eigenvalues [1]-[11] impose reduced integration step sizes, so that a complete, rigorous solution of a transient is quite impractical. Thercforc, a component representation which eliminates all fast eigenvalues atid thcrcby automatically makes the algebraic interconnection

Manuscript received September 27, 1973; revised March 27, 1974, and December 10,1974 . This work was supported by the National Research Council of Canada.

The author is with the Department of Electrical Engineering, University of Toronto, Toronto, Ont., Canada.

a valid simplification, appears to be very useful. The structure, to be considered here, of dynamic components interconnected at their terminals by an algebraic network, results as a practical model for an important class of dynamic systems.

Initially, all equations may be assumed nonlinear (or even time-varying). For the $n$ dynamic components they are

$$
\begin{aligned}
& \dot{x}_{i}=\phi_{i}\left(x_{i}, u_{i}\right) \\
& y_{i}=\psi_{i}\left(x_{i}, u_{i}\right), \quad i=1,2, \cdots, n
\end{aligned}
$$

where $x_{i}$ are vectors of state variables and $u_{i}, y_{i}$ input and output vectors, respectively. The components are interconnected through their terminal variables

$$
u=\left[u_{1} u_{2} \cdots u_{n}\right]^{T} \quad \text { and } \quad y=\left[y_{1} y_{2} \cdots y_{n}\right]^{T}
$$

by

$$
y=F(u, f)
$$

where $f$ is a forcing function. For the ensemble (1), with subscript $i$ taking all its numerical values, the more compact equations (3) can be written

$$
\begin{aligned}
& \dot{x}=\phi(x, u) \\
& y=\dot{\psi}(x, u) .
\end{aligned}
$$

Linearization of (3) and (2) produces, for the incremental variables, the following equations

$$
\begin{aligned}
& \dot{x}=A x+B u \\
& y=C \dot{x}+D u \\
& y=E u+f .
\end{aligned}
$$

It is required to obtain $u$ and $y$ as a function of time for known excitation $f$ and initial state $x(0)$.

Eliminating $y$ and $u$ from $\left(1^{\prime}\right),\left(1^{\prime \prime}\right)$, and $\left(2^{\prime}\right)$ yields

$$
\dot{x}=A^{\prime} x+B^{\prime} f
$$

where

$$
\begin{aligned}
& A^{\prime}=A-B(D-E)^{-1} C \\
& B^{\prime}=B(D-E)^{-1} .
\end{aligned}
$$

Even if the network matrix $E$ is sparse, the system matrix $A^{\prime}$ will be full and of high order. Because of the fast eigenvalues of the component matrices $A_{i}$ of the block-diagonal matrix $A$, the matrix $A^{\prime}$ will also contain fast eigenvalues 
which will seriously limit the step size when attempting to solve the system (3), (2) by a numerical integration method. For a linear problem, it might be sufficient to calculate the eigenvalues of $A^{\prime}$; this, however, is a quite difficult problem for a high-order system. Since this paper presents a proposal to overcome the above mentioned difficulties, (4) will be solved for comparison purposes only. This procedure will be termed Method 0, while the proposed approach will be termed Method 1. The basic idea of the latter consists in separately processing each component; by an implicit integration method, using step sizes large enough to eliminate the fast transients. Consequently, the equivalent system matrix $A^{\prime}$ of $\left(4^{\prime}\right)$ will also possess only slow eigenvalues and the integration can proceed in large steps to accurately describe the slowly varying system transients.

If the original problem is nonlinear and the disturbance is large, the linearization given in $\left(1^{\prime}\right),\left(1^{\prime \prime}\right)$, and $\left(2^{\prime}\right)$ has to be repeated at each step. The advantage of fast processing, due to large time steps, is maintained and is generally significant. However, for convenience of presentation, the method will be described and exemplified using, a linear system.

A special feature of the proposed method is an integration by parts with a second-order fitting for the inputs only, whereby the impulse response remains unmodified and the dynamic response of each component unaffected. This assures efficiency, accuracy, and excellent convergence. Otherwise, the method is just one of many possible implicit integration procedures. In order to assess its merits, another second-order implicit method will also be discussed (Method 2). It should be noted that this paper does not give a theoretical proof of the stability of the proposed algorithm. Good stability characteristics are plausible, however, due to the combined exponential-algebraic integrating polynomial in (5) and to the procedure of its integration by parts; also, the presented case studies and other successful applications provide confidence in the method. So far, the author did not find situations where the algorithm became unstable.

\section{Method 1: The Proposed Procedure}

The solution of $\left(1^{\prime}\right)$ for each component $i$ and the last time step $h$ is

$$
x=e^{A h} x_{n-1}+\int_{0}^{h} e^{A \tau} B u(n h-\tau) d \tau .
$$

For notational convenience subscripts $i$ and $n$ have been omitted. In (5) $u$ is approximated by a second-order expression

$$
u(n h-\tau)=a \tau^{2}+b \tau+u
$$

where

$$
\begin{aligned}
& a=\frac{1}{2 h^{2}}\left(u-2 u_{n-1}+u_{n-2}\right) \\
& b=\frac{1}{2 h}\left(-3 u+4 u_{n-1}-u_{n-2}\right) .
\end{aligned}
$$

Substitution of (6) into (5) yields

$$
x=e^{A h} x_{n-1}+\int_{0}^{h} e^{A \tau} B\left(a \tau^{2}+b \tau+u\right) d \tau .
$$

Performing by parts the integration in (8) and substituting (7) for $a$ and $b$ yields, after algebraic manipulations,

$$
x=e^{A h} x_{n-1}+L u+M u_{n-1}+N u_{n-2} .
$$

or simply

$$
x=L u+x_{p}
$$

where

$$
x_{p}=e^{A h} x_{n-1}+M u_{n-1}+N u_{n-2}
$$

stands for past effects, and $L, M, N$ are constant matrices:

$L=A^{-1}\left\{(A h)^{-2}\left(e^{A h}-U\right)+0.5(A h)^{-1}\left(e^{A h}-3 U\right)-U\right\} B$

$M=A^{-1}\left\{-2(A h)^{-2}\left(e^{A h}-U\right)+2(A h)^{-1}+e^{A h}\right\} B$

$N=A^{-1}\left\{(A h)^{-2}\left(e^{A h}-U\right)-0.5(A h)^{-1}\left(e^{A h}+U\right)\right\} B$.

In (12) the matrix $e^{A h}$ will contain zeros or negligible quantities for fast eigenvalues, if $h$ is not very small. Thus fast modes will be automatically filtered out from the output if the step $h$ is selected big enough. This assures the computational efficiency and good convergence of the new method.

\section{Alternative Procedure}

An alternate way of obtaining (10), termed Method 2, is as follows.

Denote by $w$ the integrand in (5)

$$
w(\tau)=e^{A \tau} B u(n h-\tau)
$$

and approximate it by a second-order expression

$$
w(\tau)=a \tau^{2}+b \tau+w
$$

where $a$ and $b$ have similar expressions as in (7).

Approximation (14) yields for the integral, after substituting the expressions of $a$ and $b$,

$$
\int_{0}^{h} w(\tau) d \tau=h\left(\frac{5}{12} w+\frac{2}{3} w_{n-1}-\frac{1}{12} w_{n-2}\right)
$$

where

$w=w_{n}=w(0) \quad w_{n-1}=w(h) \quad w_{n-2}=w(2 h)$.

Taking (13) into account, (15) becomes

$\int_{0}^{h} w(\tau) d \tau=h\left(\frac{5}{12} B u+\frac{2}{3} e^{A h} B u_{n-1}-\frac{1}{12} e^{2 A h} B u_{n-2}\right)$.

Substitution of (17) for the integral in (5) yields (9) and (10), where now

$$
\begin{aligned}
L & =\frac{5 h}{12} B \\
M & =\frac{2 h}{3} e^{A h} B \\
& =-\frac{h}{12} e^{2 A h} B .
\end{aligned}
$$




\section{General Algorithm}

Equation (10) is of low order, since it is written separately for each component. It is, however, valid for their ensemble. Its substitution into $\left(1^{\prime \prime}\right)$ yields

$$
y=D^{\prime} u+y_{p}
$$

where

$$
\begin{aligned}
& D^{\prime}=D+C L \\
& y_{p}=C x_{p} .
\end{aligned}
$$

From (2') and (19)

$$
\left(D^{\prime}-E\right) u=f-y_{p}
$$

which can be solved (repeatedly) for $u$ in several ways. Once $u$ is known, $y$ can be obtained from (19), and $x$ from (10).

The new value of $u$ and $x$ will then serve to update $x_{p}$ and $y_{p}$ by (11) and (20) for the next time step.

\section{Starting Procedure}

Equation (11) requires the knowledge of past values of $x$ and $u$. At the start such values are generally not available.

An initial value of $x$ and $u$ results from

$$
x_{0}=x(0)
$$

and from $\left(2^{\prime}\right),\left(1^{\prime \prime}\right)$, and (22),

$$
u_{0}=(D-E)^{-1}\left(f-C x_{0}\right) \text {. }
$$

A second value of $x$ results from

$$
x_{1}=e^{A h} x_{0}+\int_{0}^{h} e^{A \tau} B u(h-\tau) d \tau
$$

with linear approximation for $u$

$$
u(h-\tau)=u+\left(u_{0}-u\right) \frac{\tau}{h} .
$$

Substituting (24) into $\left(5^{\prime}\right)$ and performing the calculations yields $(10)$, where now

$$
x_{p}=e^{A h} x_{0}+M u_{0}
$$

and, for Method 1,

$$
\begin{aligned}
L & =A^{-1}\left\{-U+(A h)^{-1}\left(e^{A h}-U\right)\right\} B \\
M & =A^{-1}\left\{e^{A h}-(A h)^{-1}\left(e^{A h}-U\right)\right\} B .
\end{aligned}
$$

Equations (20) are now calculated using $\left(11^{\prime}\right)$ and $\left(12^{\prime}\right)$. Then (21) yields the new value of $u_{1}$, and (11') with (10) yield $x_{1}$. Thus $x_{1}, u_{0}$, and $u_{1}$ area vailable as starting values for the general algorithm (for the next step, with $n=2$ ).

For Method 2,

$$
\begin{aligned}
L & =\frac{h}{2} B \\
M & =\frac{h}{2} e^{A h} B .
\end{aligned}
$$

It is hoped that the different meaning of the subscripts in the above reasoning, as compared to Fig. 1 and some of the earlier equations, does not affect the clarity of presentation.

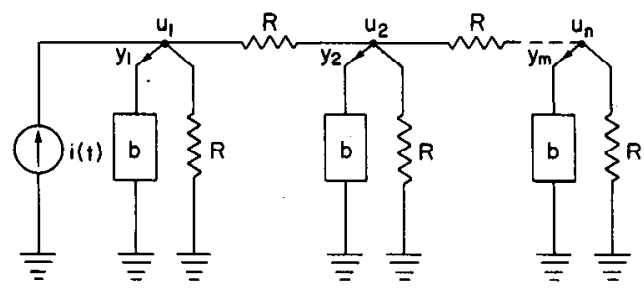

(a)

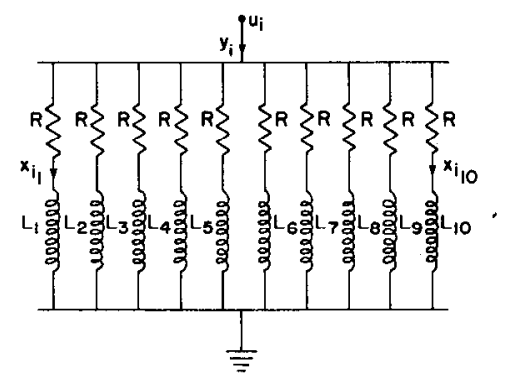

(b) $\begin{aligned} B_{i} & =\left[\begin{array}{ccc}1 & \times & 10^{-3} \\ 2 & \times & 10^{-3} \\ & \vdots \\ 5 & \times 10^{-3} \\ 6 \\ 7 \\ \vdots \\ 10\end{array}\right] \\ C_{l} & =\left[\begin{array}{llll}1 & 1 & \cdots & 1\end{array}\right], \quad D_{t}=0\end{aligned}$

$E=-\left[\begin{array}{rrrrrrr}2 & -1 & & & & & \\ -1 & 3 & -1 & & & & \\ & & & & -1 & 3 & -1 \\ & & & & & -1 & 2\end{array}\right]$,

$f=\left[\begin{array}{c}i(t) \\ 0 \\ 0 \\ \vdots \\ 0\end{array}\right], \quad u=\left[\begin{array}{c}u_{1} \\ u_{2} \\ \vdots \\ u_{n}\end{array}\right], \quad y=\left[\begin{array}{c}y_{1} \\ y_{2} \\ \vdots \\ y_{n}\end{array}\right]$

$A=\left[\begin{array}{lll}A_{1} & & \\ & \ddots & A_{n}\end{array}\right], \quad B=\left[\begin{array}{lll}B_{1} & & \\ & \ddots & B_{n}\end{array}\right]$,

$C=\left[\begin{array}{lll}C_{1} & & \\ & \ddots & C_{n}\end{array}\right], \quad D=0$.

(c)

Fig. 1. Sample network. (a) General layout; $i=$ current source, $R=1$. (b) Content of box $b$ (dynamic component $i$ of (a));

$$
L_{k}=\left\{\begin{array}{ll}
\frac{10^{3}}{k}, & \text { for } k=1, \cdots, 5 \\
\frac{1}{k}, & \text { for } k=6, \cdots, 10
\end{array} \quad R=1 .\right.
$$

(c) Matrices of (1), (2), and (3). 


\section{COMPUTER EFFORT}

Computer time and storage are both significantly reduced by increasing the step size by several orders of magnitude. The procedure, however, requires special computations, some of which are performed only once or a few times while others are repetitive. If the solution is for a nonlinear problem, then after each large step all "constants," matrices, etc., have to be recalculated, due to the linearization around the new operating point. Clearly, such updating is only worthwhile if the method assures significantly increased step sizes. For linear problems, however, the constants of (12) have to be calculated only once for a given step size. This implies matrix processing, requiring $0\left(n^{3}\right)$ operations, but $n$ is small for any particular component. Similarly, the factorization of $\left(D^{\prime}-E\right)$ in $(21)$ requires $0\left(n^{3}\right)$ operations, which is significant but has to be performed once only for a given step size. The solution of $(21)$ requires $0\left(n^{2}\right)$ operations at each step. This, however, is also required, or is implicit, in any other algorithm. Therefore, the increased step size is very much to the advantage of the proposed method.

\section{A Numerical EXAMPLE}

To illustrate the advantages of the new procedure (Method 1), presented for the calculation of transients in a large system of dynamic components with a wide range of eigenvalues, an $R L$ network was considered and solved using the new method as well as a traditional approach (Method 0 ) and the alternate implicit algorithm (Method 2). Fig. 1 gives the configuration of the sample network.

The values of $L$ were chosen to obtain two types of eigenvalues of the individual dynamic components $b$ (considered all equal, for simplicity of presentation only), producing fast and slow transients.

1) large eigenvalues, corresponding to time constants $L / R$ from 0.1 to $0.1667 \mathrm{~s}$ : the fast transients die out within $1 \mathrm{~s}$ and a quasi-steady-state will result.

2) small eigenvalues, corresponding to time constants from 200 to $1000 \mathrm{~s}$ : the slow transients will settle down to a final steady-state in about $5000 \mathrm{~s}$.

The two types of transients (fast and slow), are widely separated from each other in order to emphasize the salient features of the proposed method. Such a clear grouping of eigenvalues is very rcalistic in practical cases where the method has been applied: in dynamic system studies, the generators have widely differing eigenvalues because rotor phenomena are basically slow (due to mechanical inertia) while predominantly stator variables are fast (there are always complex eigenvalues with an imaginary part close to $377 \mathrm{rad} / \mathrm{s}$ ); in electromagnetic transient studies, transformers appear with slow variables related to core magnetization and very fast variables related to leakage fluxes and stray capacitances.

The currents through each of the $R L$ branches were chosen as state variables (see Fig. 1(b)) and, since there are 10 such branches at each node, the number of state variables is $10 \times n$ for an $n$-node network.

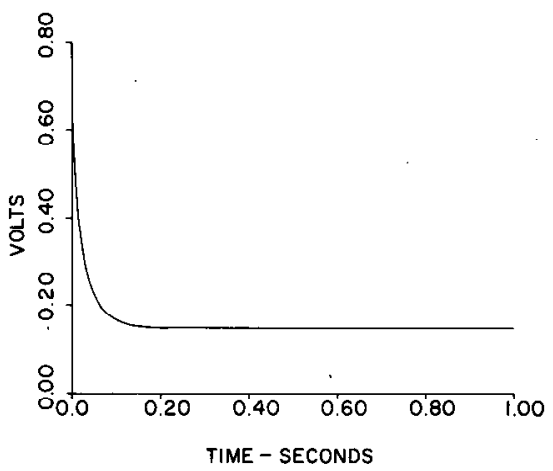

Fig. 2. Voltage at node 1 (of four nodes), with $i(t)=$ unit step; calculated with Method $0(h=0.001)$ and Method $1(h=0.01)$. The results are practically identical.

Two types of current sources $i(t)$, applied to the first node, were used as inputs $f$ to study the network response. The first was a step input ( $i=1)$, used for reasons of simplicity and next an exponentially decaying cosine function $(i=$ $\left.e^{-0.001 t} \cos 0.001 t\right)$ was used to study the response to alternating excitation. The latter was selected such that it is slow for the fast state variables but fast enough to affect the selection of a maximum possible step size.

The following observations were noted when the network transient response was calculated with each of Methods 0 , 1 , and 2, using both types of inputs described above.

\section{Method 0}

Method 0, with a fourth-order Runge-Kutta algorithm. The network was solved with various time steps $h$. All valucs of $h$ beyond $0.01 \mathrm{~s}$ gave an incorrect picture of the transients. Furthermore, for $h \geq 0.05$ the method diverged.

In order to determine the accuracy, when the network is solved by the other two methods, the Runge-Kutta solution with $h=0.001$ was taken as a basis of comparison. This could be done only for a short time interval, due to its relative computational inefficiency (see Fig. 6). Steady-state values, required to assess the quality of convergence could be obtained by direct network solution for steady-state currents and voltages.

\section{Method 1}

This method gives a very accurate result for small values of $h$, selected to study the fast transients. In particular, for $h \leq 0.01$ the results are identical to those of Method 0 with $h=0.001$ (see Fig. 2). However, if one wishes to filter out the fast modes because the interest lies in studying the slow modes only, a value of $h>1.0$ produces a direct jump, in a single step, into the quasi-steady-state, i.e., into the slow transient, which then is computed accurately.

For any step size the method converges to the correct slow-transient and steady-state values, but step sizes of the order of 0.1 , or 100 to 1000 (being too large), might not be adequate for closely following the initial part of the fast or slow transients, respectively (see Figs. 3 and 4). One single, very big step $(h>10000)$ produces directly the exact steady-state solution. For monitoring purposes the voltage 


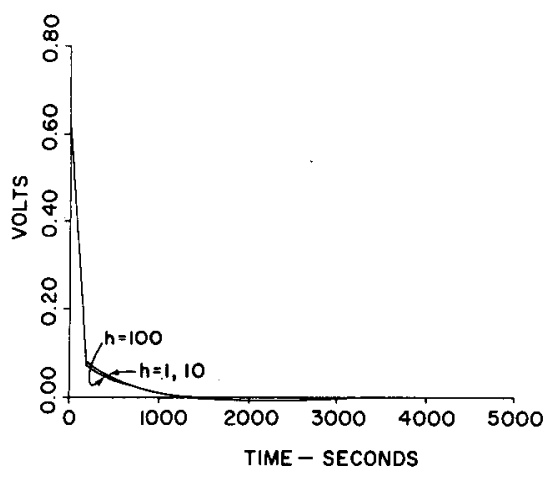

Fig. 3. Voltage at node 1 (of four nodes), with $i(t)=e^{-0.001 t} \cos 0.001 t ;$

Method 1, with $h=1,10,100$.

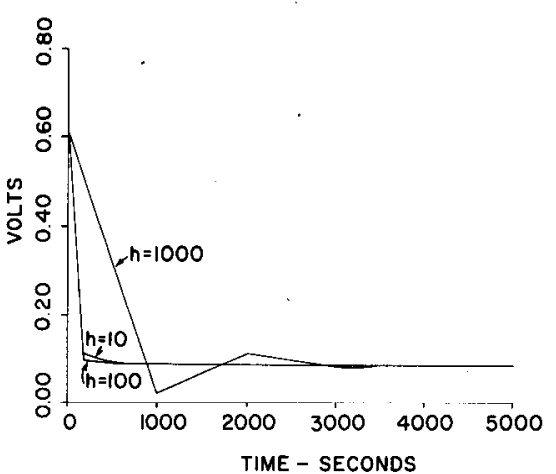

Fig. 4. Voltage at node 1 (of twenty nodes), with $i(t)=$ unit step; Method 1, with $h=10,100,1000$. All results converge to the same steady-state value.

on bus 1 has been represented in all cases, but all basic variables have been calculated.

If one wishes to study both the fast and the slow portions of the transient, then it is feasible to use a small step size ( $h \leq 0,01$ ) up to, say, $20 \mathrm{~s}$ of the transient and then switch to $h=10$ for its slow part, up to steady-state. Such a procedure is easy to program, since no precautions are needed to assure convergence, and gives an accurate picture of both fast and slow modes, with very significant savings in computer time and storage.

\section{Method 2}

For small step sizes $(h \leq 0.01)$ this method gives very accurate results, but for $h>0.01$ the results in the transient state are incorrect and the program converges to an incorrect solution in steady-state. This behavior is emphasized in Fig. 5. Therefore, this method is inferior to the previous one, even though it is slightly simpler in its algebraic formulations.

The results of all methods tested are summarized in Table $I$ and in Figs. 6 and 7. These, combined with the excellent convergence of Method 1, clearly demonstrate its computational superiority. It should be noted that all programming was done in double precision, in order to ascertain that lack of convergence was not the result of numerical instability. The programs were run on IBM 370/165 and Figs. 2-5 were computer drawn (CALCOMP).

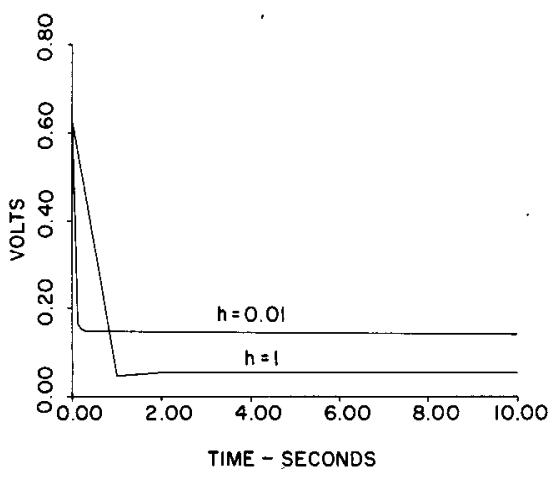

Fig. 5. Voltage at node 1 (of four nodes), with $i(t)^{\circ}=$ unit step Method 2 , with $h=0.01 \rightarrow$ will converge to correct value and $h=1 \rightarrow$ will converge to incorrect value.

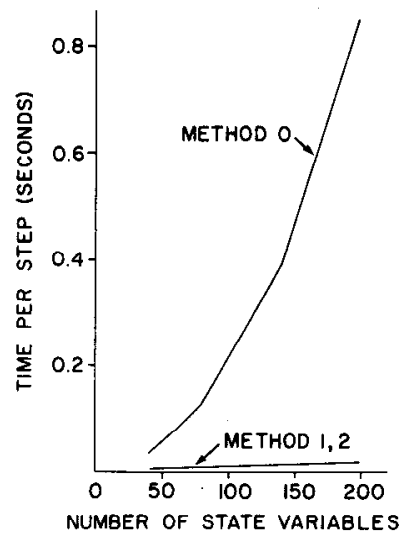

Fig. 6. Computer time per iteration step.

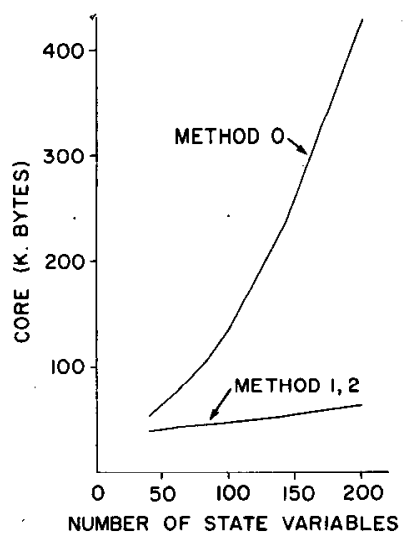

Fig. 7. Computer storage requirements.

\section{OTHER TESTS}

In the previous example, the proposed method was compared with a fourth-order Runge-Kutta algorithm because the wide spread acceptance of the latter for the numerical solution of ordinary differential equations. There are, however, special methods for the solution of stiff differential equations [12], [13] and an application of the proposed method on power system dynamics was performed and compared with the high-order Crank-Nicholson method (HCN5) [4] which proved to be faster than the well-known Gear's algorithm [7] in solving stiff differential equations.

With a system of 31 state variables, the proposed implicit integration method required approximately 20 percent less 
TABLE I

COMPuTER Requirements for ANy STEP Siże

\begin{tabular}{|c|c|c|c|c|c|c|}
\hline \multirow{2}{*}{$\begin{array}{l}\text { No. of } \\
\text { State } \\
\text { Variables }\end{array}$} & \multicolumn{2}{|c|}{$\begin{array}{l}\text { AETHOD O } \\
\text { (Runge Kutta) }\end{array}$} & \multicolumn{2}{|c|}{ METHOD I } & \multicolumn{2}{|c|}{ METHOD 2} \\
\hline & $\begin{array}{l}\text { Memory } \\
\text { Usage } \\
\text { (K.Bytes) }\end{array}$ & $\begin{array}{l}\text { Time per } \\
\text { step } \\
\text { (Seconds) }\end{array}$ & $\begin{array}{l}\text { Memory } \\
\text { Usage } \\
\text { (K.Bytes) }\end{array}$ & $\begin{array}{l}\text { Time per } \\
\text { step } \\
\text { (Seconds) }\end{array}$ & $\begin{array}{l}\text { Memory } \\
\text { Usage } \\
\text { (K.By'tes) }\end{array}$ & $\begin{array}{l}\text { Tine } \\
\text { per step } \\
\text { (scconds) }\end{array}$ \\
\hline 40 & 52 & 0.033 & 38 & 0.0021 & 38 & 0.0022 \\
\hline 60 & 74. & 0.078 & 42 & 0.0033 & 40 & 0.0033 \\
\hline 80 & 102 & 0.129 & 44 & 0.0047 & 42 & 0.0048 \\
\hline 100 & 136 & $0: 213$ & 46 & 0.0070 & 48 & 0.0067 \\
\hline 140 & 232 & 0.387 & 52 & 0.0096 & 50 & 0.0093 \\
\hline 200 & 430 & 0.851 & 62 & 0.0161 & 60 & 0.0160 \\
\hline
\end{tabular}

computer core and was 50 percent faster than HCN5. It was found that as the power system gets more complex the computer time and core requirement for the new method increase at a slower rate than for HCN5. Since eigenvalue analysis of such high-order stiff systems is computationally extremely difficult, the described procedure appears to be of practical significance for dynamic studies in large power systems.

Another field of fruitful applications proved to be the calculation of electromagnetic transients in high-voltage systems [14]. This is done by following the wave propagation along lossy transmission lines with ground return. For line modes the wave fronts are quite steep which would normally impose a very small integration step. The implicit integration method (slightly modified to permit consideration of travel time-delays) permitted, however, to increase the time step, after the overvoltage of interest has sufficiently attenuated, and thus obtain quite rapidly the steadystate condition after switching.

\section{CONCLUSIONS}

Large dynamic systems built up by algebraically interconnected components which have a wide range of eigenvalues can be advantageously solved by the new implicit method described in the paper. The method takes advantage of a procedure of integrating by parts the dynamic equations of the components.

The result is excellent convergence, fast and accurate solution, with low core requirements. The computer time and storage increase slowly and approximately linearly with problem size. These favorable results are due to the specific problem nature and formulation, resulting in strong diagonal dominance and permitting sparse matrix techniques. Without these characteristics, the method would lose its efficiency.

The natural applications of the method are in systems of interconnected dynamic components, e.g., power systems. Applications in both dynamic and electromagnetic transients have been successfully tested, and will be described separately.

\section{ACKNOWLEDGMENT}

The author wishes to thank Z. S. Pervaiz for performing the numerical studies with care and competence.

\section{REFERENCES}

[1] C. F. Curtiss and J. O. Hirschfelder, "Integration of stiff differential equations," Proc. Nat. Acad. Sci. U.S., vol. 38, pp. 235-243, 1952.

[2] H. H. Rosenbrock, "Some general implicit processes for the numerical solution of differential equations," Comput. J., vol. 5, pp. 329-330, 1962.

[3] C. E. Treanor, "A method for the numerical integration of the coupled first order differential equations with greatly different time constants," J. Math. Comput., vol. 20, pp. 39-45, Jan. 1966.

[4] E. J. Davison, "A high order Crank-Nicholson technique for solving differential equations," Comput. J., vol. 10, pp. 195-197, Aug. 1967.

[5] M. E. Fowler and R. M. Warten, "A numerical integration technique for ordinary differential equations with widely separated eigenvalues," IBM J. Res. Develop., vol. 11, pp. 537-543, 1967.

[6] M. R. Osborne, "A new method for the inlegration of stiff systems of ordinary differential equations," in Proc. IFIP Congr., pp. 200-204, 1968.

[7] C. W. Gear, "The automatic integration of stiff ordinary differential equations," in Proc. IFIP Congr., pp. 187-193, 1968.

[8] - "The automatic integration of ordinary differential equations," Commun. Ass. Comput. Mech., vol. 14, pp. 176-179, Mar. 1971.

[9]. H. Schichman, "Integration system of a nonlinear network analysis program," IEEE Trans. Circuit Theory, vol. CT-17, pp. 378-386, Aug. 1970.

[10] R. Oswald and O. J. M. Smith, "Integration of nonlinear differential systems with wide. eigenvalue range," IEEE Trans. Power App. Syst., vol: PAS-90, pp. 2586-2589, Nov./Dec. 1971 .

[11] G. Bjurel, G. Dahlquist, B. Lindberg, and S. Linde, "Survey of stiff differential equations," Dep. Inform. Processing Comput. Sci., Royal Inst. Tech., Stockholm, Sweden, Rep. NA 70-11.

[12] C. W. Gear, Numericál Initial Value Problems in Ordinary Differential Fquations. Englewood Cliffs, N.J.: Prentice-Hall, 1971.

[13] S. W. Director, Computer-Aided Circuit Design: Simulation and Optimization. Stroudsburg, Pa.: Dowden, Hutchinson and Ross, 1973.

[14] A. Semlyen and A. Dabuleanu, "Fast and accurate switching transient calculations on transmission lines with ground return using recursive convolutions," IEEE Trans. Power App. Syst., vol. PAS-94, pp. 561-571, Mar. 1975.

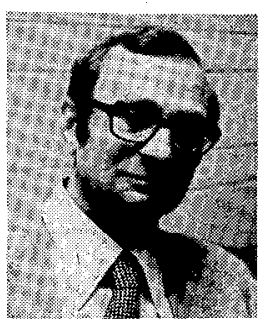

Adam Semlyen (SM'70) received the Dipl. Eng. honors degree in electrical engineering from the Polytechnic Institute of Timisoara, Timisoara, Rumania, and the Dr. Eng: degree from the Polytechnic Institute of Iasi, Iasi, Rumaniâ:

He started his career with the Central Power Station of Timisoara where he was primarily engaged in testing of power equipment, meters and relays. Simultaneously, he was on the staff of the Faculty of Electrical Engineering of the Polytechnic Institute of Timisoara, teaching a wide range of courses in power engineering. His research interest was in current transformers, power system analysis, and overvoltages in high-voltage systems. As a Consultant to the Regional Power 
Authority he designed equipment for compensation of capacitive ground currents. In 1969 he was with Benedikt and Jager, in Vienna, Austria, where he did development studies in reactors and special transformers. He then joined the University of Toronto, Toronto, Ont., Canada, where he is Professor in the Department of Electrical
Engineering. His current interest is in power system dynamics and electromagnetic transients in transmission lines and systems. He has written a textbook and papers in power systems and related topics.

Dr. Semlyen is a member of the Association of Professional Engineers of the Province of Ontario and a member of CIGRE.

\section{Letters to the Editor}

\author{
Relation Between the Network Determinants \\ MIRKO M. MILIĆ
}

\begin{abstract}
The relation is established between the determinant of the network equations for branch variables and the determinant of the cutset admittance matrix or the determinant of the loop impedance matrix for general linear, lumped, time-invariant networks composed of a finite collection of multiterminal elements.
\end{abstract}

\section{INTRODUCTION}

As is known, the determinant of the matrix of the network equations for the branch variables, consisting of Kirchhoff and terminal equations, framed as a set of algebraic and first-order differential equations det $\boldsymbol{H}(s)$, determines the natural frequencies of the network [1]. Since in most cases the network elements have an immittance (impedance or admittance) terminal description, or they can be grouped in composite multiterminal elements (subnetworks) having such a description, when one considers the order of complexity, it is advantageous to relate det $\boldsymbol{H}(s)$ to the determinant of the cutset admittance matrix det $Y_{n}(s)$, or to the determinant of the loop impedance matrix det $\boldsymbol{Z}_{m}(s)$. The relation between $\operatorname{det} \boldsymbol{H}(s)$ and $\operatorname{det} \boldsymbol{Y}_{n}(s)$ has been derived for $R L C$ networks [1], for $R L C$ networks containing dependent current sources [2] and for $R C$ networks containing gyrators [3]. Relations among the network determinants and the determinants of the coefficient matrices appearing in the state equations for $R L C$ networks have also been obtained [4].

The purpose of the work reported here is to derive the relation linking det $\boldsymbol{H}(s)$ and det $\boldsymbol{Y}_{n}(s)$ or det $\boldsymbol{Z}_{m}(s)$ for the general case of linear, time-invariant networks made of an interconnection of arbitrary multiterminal elements, each of which possesses an admittance or an impedance terminal characterization. The distinctive feature of this case is that the terminal equations involve rational matrices rather than first-order polynomial matrices as required by the network equations in question.

Manuscript received June 6, 1974; revised November 11, 1974.

The author is with the Faculty of Electrical Engineering, University of Belgrade, Belgrade, Yugoslavia.

\section{THE NETWORK EQUATIONS}

Consider a network composed of a finite collection of multiterminal elements, each multiterminal element being described by its terminal equations pertaining to a terminal graph [5]. Let the network graph obtained by adjoining the terminal graphs of the individual multiterminal elements be connected and have $b$ branches and $n$ nodes. Denote by $I(s), V(s), J(s)$, and $E(s)$ the Laplace transforms of branch current vector, branch voltage vector, cutset current generator vector, and loop voltage generator vector, respectively, and denote by $Q$ the $(n-1) \times b$ independent cutset matrix and by $\boldsymbol{B}$ the $(b-n+1) \times b$ independent loop matrix. Then Kirchhoff equations are written

$$
\begin{aligned}
& Q I(s)=\left[\begin{array}{ll}
Q_{1} & Q_{2}
\end{array}\right]\left[\begin{array}{l}
I_{1}(s) \\
I_{2}(s)
\end{array}\right]=J(s) \\
& B V(s)=\left[\begin{array}{ll}
B_{1} & B_{2}
\end{array}\right]\left[\begin{array}{l}
V_{1}(s) \\
V_{2}(s)
\end{array}\right]=E(s)
\end{aligned}
$$

where matrices and vectors with subscripts 1 and 2 refer to the tree and cotree, respectively.

The setting of the network equations for the branch variables in the form of a set of algebraic and first-order differential equations requires the terminal equations being expressed in the same form. Thus starting from a hybrid terminal description

$$
\begin{aligned}
{\left[\begin{array}{l}
I_{1}(s) \\
V_{2}(s)
\end{array}\right] } & =H_{e}(s)\left[\begin{array}{l}
V_{1}(s) \\
I_{2}(s)
\end{array}\right] \\
& =\left[\begin{array}{ll}
H_{11}(s) & H_{12}(s) \\
H_{21}(s) & H_{22}(s)
\end{array}\right]\left[\begin{array}{c}
V_{1}(s) \\
I_{2}(s)
\end{array}\right]
\end{aligned}
$$

with $\boldsymbol{H}_{e}(s)$ a $b \times b$ rational matrix of the most general form

$$
\boldsymbol{H}_{e}(s)=\boldsymbol{H}_{e 0}(s)+J_{e}(s)
$$

where $\boldsymbol{H}_{e 0}(s)$ is zero at infinity and $\boldsymbol{J}_{\boldsymbol{e}}(s)$ is a matrix polynomial

$$
J_{e}(s)=J_{0}+J_{1} s+J_{2} s^{2}+\cdots+J_{q} s^{q}
$$

${ }^{1}$ It is well known that higher order poles at infinity may occur in active networks. 